\title{
Star Formation Under Cosmological Conditions
}

\author{
Mounib F. El Eid ${ }^{1}$ \& Cynthia Saad ${ }^{1}$ \\ ${ }^{1}$ American University of Beirut Department of physics, meid@aub.edu.lb, crs07@mail.aub.edu.lb
}

\begin{abstract}
The first stars have transformed the universe from the infrared background to the light. Thereby, this so called "dark age" ended by re-ionization through the ultraviolet light of the first stars. A short overview is given about the cosmological connection of the formation of the first stars in the universe. The physical background underlaying the process of their formation is outlined and their basic properties are described. Preliminary results of computational simulations are presented.
\end{abstract}

\section{Introduction}

The study of star clusters reveals curious difference between the youngest and the oldest stars in the galaxy, where two star populations are observed. Pop I stars are metal-rich showing strong spectral lines of heavy elements like in the sun (metals is described by metallicity that is nuclear species beyond Lithium). The population II stars are metal-poor with weak spectral lines of heavy elements.

A question is why two distinct populations. To answer this question, we have to go back to the first minutes after big bang to discover that the universe was not able to produce elements beyond the element Lithium (see [13] and references therein). Another question is: how did the survived pop II stars with the smallest masses get their metals. Clearly from previous generation of stars. The concept of two populations provides insight of our origin, since the He-fusion in red giant stars produced the element ${ }^{12} \mathrm{C}$ involved on our DNA.

The Pop II stars found in globular clusters cannot be the first stars to have been formed after big bang, because these first stars were mainly $\mathrm{H} / \mathrm{He}$ stars and can be termed "zero-metal stars". Thus, pop III stars would have been formed in gas clouds composed mainly of $\mathrm{H} / \mathrm{He}$. Such clouds have higher pressure than the metal-rich clouds of the same temperature. This may already indicate formation of high-mass stars of several hundred solar masses [9].

Despite of great effort, the discovery of pop III stars remains elusive. According to [1] the star SMSSJ031300-670839.3 has a metallicity of Fe/H] $<-7.1$ (about $10^{-9}$ solar and no iron, but enhancement in carbon and magnesium [2]. However, indirect evidence of Pop III stars exists through the infrared images of the Spitzer telescope which reveal a distant infrared background from pop III stars. Another indication is related to the re-ionization of the universe. A very massive pop III star can reach effective temperature of $10^{5} \mathrm{~K}$ and their luminosity approaches the Eddington limit [3]:

$$
L=1.3 \times 10^{40}\left(M / M_{\odot} \mathrm{erg} / \mathrm{s}\right.
$$

Clearly, $\mathrm{L}$ is proportional $\mathrm{M}$, or the luminosity and color depend on the mass. The ultraviolet photons from these stars are able to re-ionize the very abundant hydrogen atoms in the universe. This happened after the dark age, so that the popIII stars have transferred the universe 
from darkness into light. The question is when did that dark age end ? We mention recent observations below.

So far, We may conclude that the enrichment of heavy elements up to the iron group elements by supernova explosions has the effect that subsequent star formation occurs in clouds of lower internal pressure such that lower-mass star can be formed.

When did the dark age end? Recent observations and their interpretation [4] indicate that the dark age ended about 180 million years after the recombination time of $387000 \mathrm{yrs}$ and this is by about a factor of two shorter than previously thought. Their argument is that the UV light from early stars penetrates the primordial hydrogen gas and alters the excitation state of the $21-\mathrm{cm}$ hyperfine structure line. This allows the absorption of photons from the microwave background radiation. The spectral distortion is expected to be observed today at radio frequency of less than $200 \mathrm{MHZ}$. The observed profile was centered at $78 \mathrm{MHZ}$. Such observations will have an impact on the initial conditions involved in the process of the first star formation.

\section{The formation Scenario of the Early Stars}

The first question is: How did the universe evolve from extreme uniformity into a complex structure we see today?. The observations of microwave isotropy data [5] indicate that the universe was extremely uniform, but with spatial fluctuations in the energy density and gravitational potential of about $10^{-5}$. Such fluctuation generated in the early universe due to gravitational instability led to the formation of galaxies. The small fluctuation can be treated as linear perturbation and this represents a powerful probe of the last scattering surface at redshift of $\mathrm{z} \sim 1100$. Complementary observations came from high-redshift quasars at $\mathrm{z} \sim 5$, $10^{9} \mathrm{yr}$ after big bang. Not much is known about the epoch at $\mathrm{z}=1100$ to 5 , which is about the period of the dark age.

The Hierarchical scenario of structure formation is specified by cold dark matter (CDM) models, which suggest that the collapse of first baryonic objects is expected at $\mathrm{z} \sim 50$ to 10 involving dark matter halo of mass about $10^{6} \mathrm{M}_{\odot}$ comparable to the cosmological Jean's mass [6]. Next question is: How do we know that the universe undergone re-ionization by first stars?. This is indicated by the absence of the absorption feature in the spectra of redshiftquasars at $\mathrm{z}>5$ (see [7]). The UV photons from the first stars are expected to have re-ionized the universe.

Another question is: How to probe when the formation of the first stars occurred despite the lack of observations. Or, observations at high redshift are needed. This is the main focus of the next generations of telescopes (NGST), (see http;//ngst.gsf.nasa.gov). The NGST will probe the UV emission from source with $\mathrm{z}>10$, will help to determine the geometry of the universe, and will be able to help studying the effects of dark matter.

An interesting remark is how can we understand the primordial star formation in principle. Several reasons to think that the early star formation was relatively simpler than its presentday star formation since:

(a) The small perturbation of the uniform cosmological background can be adopted provided the so called $\Lambda C D M$ model remained valid on small scale (see basics of this model are presented below).

(b) Absence of heavy elements leaves the atomic and molecular hydrogen as main agent of radiative cooling and source of opacity.

(c) The intergalactic medium had no source of turbulent motion before supernova explosions

(d) The magnetic field might be less efficient, but still not is not sufficiently explored,

Despite these arguments, it remains challenging to consider processes occurring from cosmological scale to proto-stellar scale. 


\section{Dark Matter}

Dark matter is characterized by negligible velocity dispersion, where candidate could be rather massive photions(the hypothetical supersymmetric counterpart of the photons), or the invisible axions. The $\Lambda \mathrm{CDM}$ mode has the following average density parameters: $\Omega_{m}=0.29$ (cold dark matter density parameter), $\Omega_{b}=0.047$ (baryon density parameter), $\Omega_{\Lambda}=0.71$ (dark energy density parameter), $H_{0} / 100=0.72$ (present Hubble constant over 100 ). The $\Omega$ 's are normalized to the critical density of the universe given by

$$
\rho_{c}=3 H_{0}^{2} /(8 \pi G)
$$

for $H_{0}=73 \mathrm{~km} / \mathrm{s} / \mathrm{Mpc}, \rho_{c}=1.0 \times 10^{-26} \mathrm{~kg} / \mathrm{m}^{3}$. Precise measurements of the CMB ([5]) have helped to confirm that we live in a flat universe with these parameters.

The primordial star formation is unique, since it is related to CDM model of cosmology. This seems to have happened in a minihalo of about $10^{6} \mathrm{M}_{\odot}$ at redshifts $\mathrm{z}=20-30$ ([3]). The James Webb Space telescope (JWST) will give information about the abundances of the most metal-poor stars, which will constrain the nucleosynthesis from the first supernovae.

\section{Chemistry and Cooling}

How is the cooling of the gas cloud mediated?. A useful review about the chemical species involved in cooling of the gas cloud is given by [8]. The collapse of the minihalo may be approximated by a state of virial equilibrium. Simple calculation([9]) show that the virial temperature is

$$
T_{v i r} \simeq 2.0 \times 10^{3}\left(M_{h} / 10^{6} \mathrm{M}_{\odot}\right)^{2 / 3}(1+z) / 20
$$

This indicates that typical gas temperature in minihalos are below the critical temperature of about $10^{4} \mathrm{~K}$ needed for efficient cooling via atomic hydrogen. This is important, since if the gas were unable to cool there would be no further collapse and no fragmentation. In a low temperature gas, the cooling is effective due to $\mathrm{H}_{2}$ molecules, However the formation of these molecules in the early universe is not effective. The reason is the following. The hydrogen molecule posses a high symmetry, since it has two identical atoms and does not have a permanent electric dipole moment. Radiative transition can only occur via slow magnetic quadrupole radiation. If two hydrogen atoms collide they form short-lived compound and cannot radiate their excess of kinetic energy and decay back.

In interstellar medium (ISM), dust grains provide surfaces for the H-atoms too meet and provide also a sink for the binding energy that must be liberated to enable the formation of the molecules. Since no dust grains exist in a primordial gas, the reaction $\mathrm{H}+\mathrm{H} \rightarrow \mathrm{H}_{2}+\gamma$ does not occur unless one of the $\mathrm{H}$-atom is in an excited state which is rather unlikely. Other possibilities are:

(i) in case of high particle density $\mathrm{n}>10^{8} \mathrm{~cm}^{-3}$, three-body reactions are possible (see [10] and references therein) such as

$\mathrm{H}+\mathrm{H}+\mathrm{H} \rightarrow \mathrm{H}_{2}+\mathrm{H}$

$\mathrm{H}+\mathrm{H}+\mathrm{H}_{2} \rightarrow \mathrm{H}_{2}+\mathrm{H}_{2}$.

(ii) for lower particle density $\mathrm{n}<10^{8} \mathrm{~cm}^{-3}$, reactions are

$H+e^{-} \rightarrow H^{-}+\gamma$

$H^{-}+H \rightarrow H_{2}+e^{-}$

These are mediated by the ion $\mathrm{H}^{-}$, where the free electrons catalysts are relic from the epoch of recombination at redshift $\mathrm{z}=1100$. Other set of reactions are mediated by $\mathrm{H}^{+}$:

$H+H^{+} \rightarrow H_{2}^{+}+\gamma$

$\mathrm{H}_{2}^{+}+\mathrm{H} \rightarrow \mathrm{H}_{2}+\mathrm{H}^{+}$ 
It is clear that the presence of free electrons and protons lead to the formation of the $\mathrm{H}_{2}$. But, why is the formation is efficient despite the low gas temperature?. The answer is that the intergalactic medium (IGM) was not in ionization equilibrium. It has retained a fractional ionization of about $2 \times 10^{-4}$ from the epoch of recombination and this is because the Hubble expansion ensured that the recombination time scale exceeded the expansion time scale before the IGM can reach ionization equilibrium.

\section{Some Results from current Simulations}

The following preliminary results are part of the $\mathrm{PhD}$ project of Mrs Cynthia Saad. The input quantities used in these simulations are the following CDM-parameters: $\Omega_{\Lambda}=0.728$, $\Omega_{m}=0.242, \Omega_{b}=0.045, H_{0} / 100=0.704$. In addition, the mass of the minihalo is taken as $M_{h}=1.65 \times 10^{6} \mathrm{M}_{\odot}$, the central baryon particle density $n_{b}=10^{8} \mathrm{~cm}^{-3}$, the redshift was taken as $\mathrm{z}=23.43$ and the spectral index $\sigma_{s}=0.96$.

Briefly, the computational tools used is a modified 3d-MHD hydrodynamic code due to [12] and the chemistry network used is due to [11]. However, the following results did not include the magnetic field yet, thus they are results of hydrodynamical calculations(briefly: HD calculations).

In Fig. (1), left panel, the formation of the $\mathrm{H}_{2}$ molecules is illustrated . In the density range as shown in the Figure, the production of the $\mathrm{H}_{2}$ molecules is mediated by the $\mathrm{H}^{-}$ion, which itself requires the existence of free electrons. Furthermore, the $\mathrm{H}^{+}$plays a role in this production (see sect. 4). However, at high density exceeding $10^{8} \mathrm{~cm}^{-3}$, three-body reactions will contribute as we have argued in sect. 4.

Fig. (1) on the right panel, shows the temperature variation with the following characteristics: at low density, the gas is heated up through adiabatic compression until the maximum at a temperature exceeding $1000 \mathrm{~K}$ is reached, which resembles the virial the temperature of the host minihalo. Afterward, the gas begins to cool through vibrational transitions of the $\mathrm{H}_{2}$ molecules, so that a minimum temperature is reached near $100 \mathrm{~K}$ and at a density close to $\mathrm{n} \simeq 10^{4} \mathrm{~cm}^{-3}$

In Fig. (2) a simulation shows the protostar formation (left panel), while the right panel shows the formation of an accretion disk around the protostar. More extended calculations are needed to find out the final mass of the protostar. This work is in progress (C. Saad et al. in preparation).

\section{Summary}

- Understanding the formation of the first stars is an exciting subject connecting star formation with cosmology.

- Primordial star formation is unique, since initial conditions can be related to the cold dark matter of cosmological structure formation (see sect. 2).

- The investigation in this field will motivate the work of the next generation observational facilities to probe the high-redshift universe.

- The mass range of the primordial stars will give insight about the initial enrichment of the heavy elements beyond Lithium.

- It is challenging to figure out how the so called "dark age" ended by the emergence of the first stars. It appeals to the imagination. 

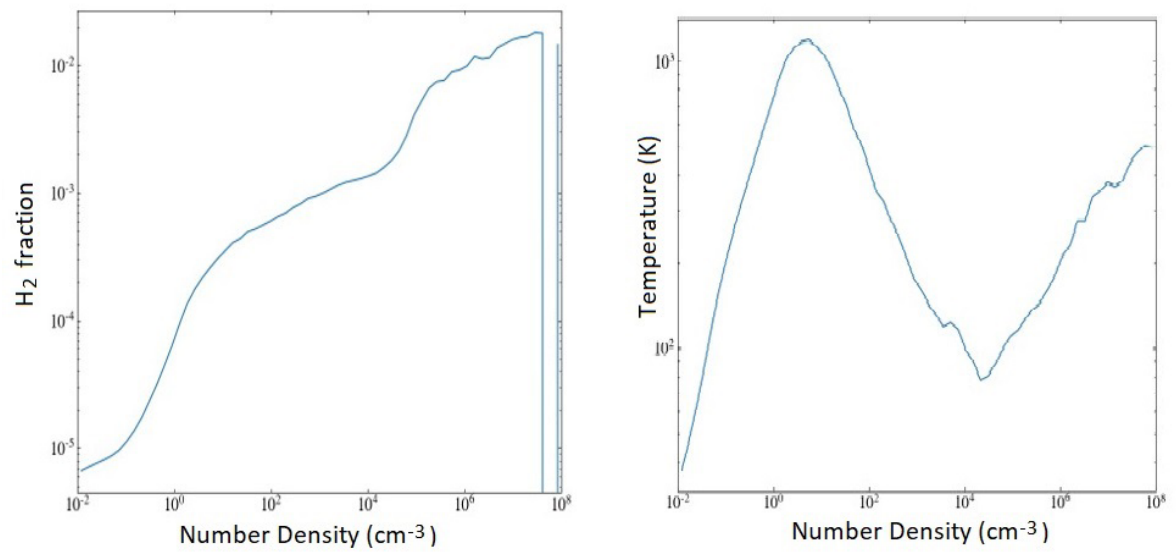

Figure 1. The Figure on the left shows the fraction of the formed $\mathrm{H}_{2}$ molecule versus number density. The Figure on the right displays the behavior of the temperature versus number density, see text.
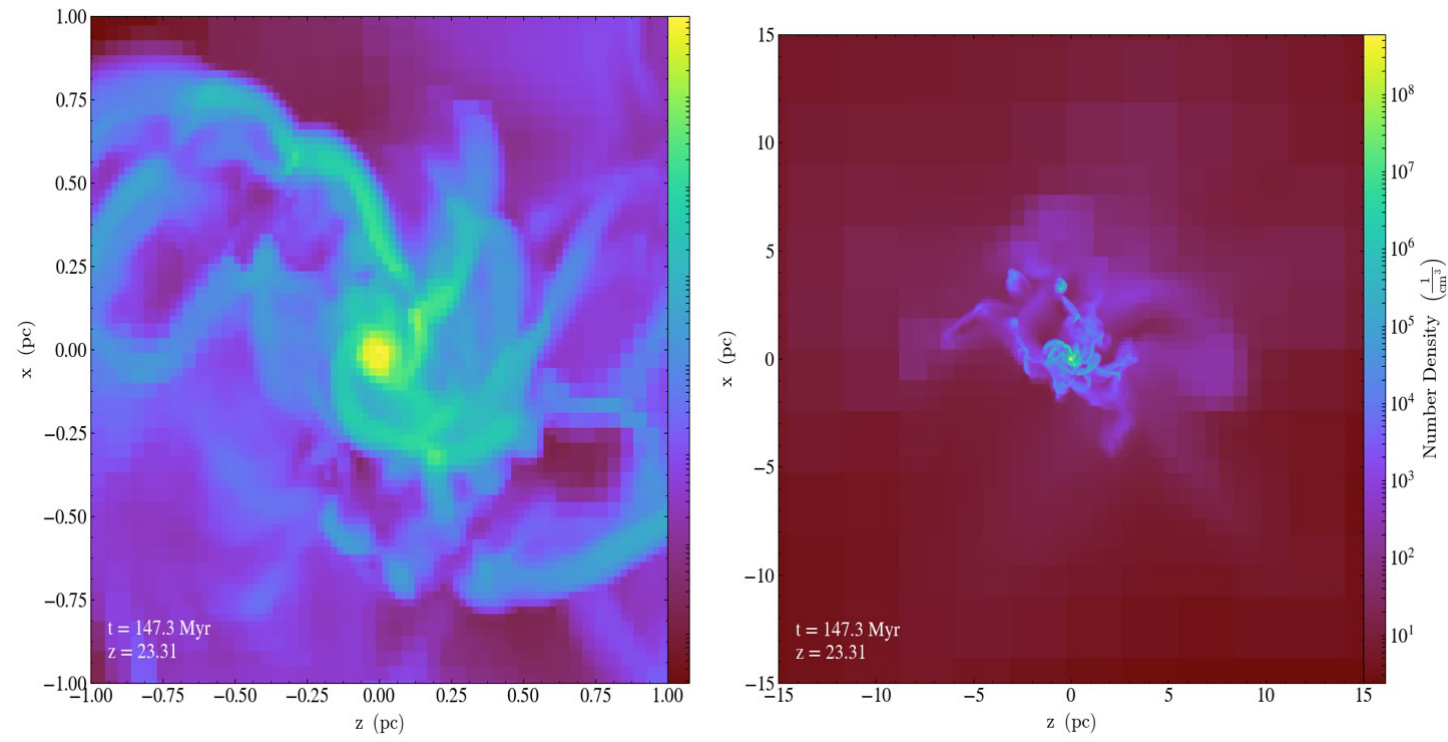

Figure 2. Formation of a protostar in a minihalo. The left figure shows a protostar in formation, while the left figure shows an accretion disk surrounding the protostar. These Figures are snapshots at the indicated time at the given redshift

\section{Acknowledgment M.F. El Eid thanks the conference organizer for a partial support.}

\section{References}

[1] Keller, S.C. et al., Nature, 506, 463 (2014) 
[2] Bessel,M.S. et al., APJL, 806, L16 (2015)

[3] Loeb, A., How did the First Stars and Galaxies Form? Princton Universitry Press, Princton, New Jersey, 2010, p. 74

[4] J. D. Bowman, J.D., et al. Nature, 555, 67 (2018)

[5] Bennett, J.D., et al. APJL, 464, 1 (1996)

[6] Tegmark, M. et al., APJ, 474, 1 (1997)

[7] Gunn, J.E. \& Peterson, B.A., APJ, 142, 1633 (1965)

[8] Glover, S.C.O, \& Abel, T., MNRAS, 388, 1627 (2008)

[9] Bromm, V., Reports on Progress in Physics, 76, 112901 (2013).

[10] Glover, S., Space Sci. Rev., 117, 445 (2005)

[11] Smith, B. D., et al. MNRAS, 466, 2217 (2016)

[12] Bryan, G. L., et al. ApJS, 211, 19 (2014)

[13] Makki,T. El Eid, M., Mathews, G., Modern Physics Letters A, 23, 1950194 (2019) 